\title{
El método clínico como método de enseñanza pedagógica
}

\section{The clinical method as a pedagogical teaching method}

\section{O método clínico como método de linguagem pedagógica}

\author{
Nancy A. Sorroza-Rojas I \\ nancysorroza@uees.edu.ec \\ José P. Barberan-Torres II \\ pbarberan@uees.edu.ec \\ Nancy V. Cajas-Flores ${ }^{\text {III }}$ \\ ncajasf@uees.edu.ec \\ Jesús E. Rodríguez-Villacis IV \\ jesusrodriguez@uees.edu.ec \\ Bolívar E. Jinez-Sorroza V \\ kikejinez@gmail.com \\ García L. Frella-Soraya VI \\ soraya.garcial@ug.edu.ec
}

Recibido: 30 de enero de 2017 * Corregido: 20 de febrero de 2017 * Aceptado: 20 junio de 2017

I. Universidad de Especialidades Espíritu Santo, Samborondón, Ecuador.

II. Universidad de Especialidades Espíritu Santo, Samborondón, Ecuador.

III. Universidad de Especialidades Espíritu Santo, Samborondón, Ecuador.

IV. Universidad de Especialidades Espíritu Santo, Samborondón, Ecuador.

v. Universidad de Guayaquil, Guayaquil, Ecuador.

VI. Universidad de Guayaquil, Guayaquil, Ecuador. 


\section{Resumen}

El método clínico, método para la ayuda médica a personas enfermas, forma una categoría fundamental en las ciencias clínicas. Su uso como método de enseñanza en las disciplinas y asignaturas del grupo de la profesión, enmarcadas en el ciclo clínico del área médica, contribuye no sólo al mayor dominio de dicho método, sino también a la sistematización de todas aquellas habilidades que, en forma de sistema, se integran en la ejecución de este. Por ello, el método clínico constituye la vía fundamental para la formación y desarrollo de las habilidades profesionales; y se evidencia que la lógica de la profesión se convierte en la lógica del proceso de formación del médico general integral básico. Este artículo tiene como propósito fundamentar la consideración del método clínico como método para la enseñanza y aprendizaje de las habilidades profesionales del médico.

Palabras clave: Método clínico; método de enseñanza; aprendizaje. 


\begin{abstract}
The clinical method applied in the medical assistance to sick people is an essential category in the Clinical Sciences. Its use as a method in the teaching learning process in the disciplines and subjects of the clinical cycle in the medical profession contributes not only to master this method deeply, but to systematize all those abilities, that as a system, gather as a whole during its application. Therefore, the clinical method constitutes the main via to develop the student?s professional abilities, proving that the logic of the profession becomes at the same time the logical thinking of the Comprehensive General Doctor?s formative process. This paper?s main purpose is to consider the clinical method as the teaching method for teaching, learning and developing the doctors? professional abilities
\end{abstract}

Key words: Clinical method; teaching method; learning. 


\section{Introducción.}

Un aspecto relevante de la preparación científico-técnica de los educandos radica en el conocimiento y dominio del método clínico, el cual constituye el método de trabajo del facultativo para la atención a individuos enfermos (independientemente del campo o rama de la Medicina en que el profesional realice su labor) complementado por el método epidemiológico para la proyección poblacional de su trabajo asistencial. (Dupuy F \& Rodríguez Rivera, 1990)

Existe una crisis del método clínico que tiene consecuencias preocupantes en la profesión médica que afecta a todos, países desarrollados y subdesarrollados. Esta crisis gira sobre todo alrededor de los siguientes aspectos: deterioro de la relación médico paciente, menosprecio del valor del interrogatorio y del examen físico y sobrevaloración de la función de la tecnología. (Espinosa Brito, 2010)

Este artículo de revisión tiene como objetivo fundamentar cómo, adicionalmente a su consideración como un contenido de aprendizaje con categoría de invariante del conocimiento, el método clínico es el principal método para la enseñanza y aprendizaje de las habilidades profesionales vinculados al área medica.

\section{Desarrollo Teórico.}

El método, como categoría, se refiere a la manera determinada de procedimientos para ordenar la actividad con el fin de lograr un objetivo; o sea, el método describe la dinámica del proceso, la vía para su ejecución, la lógica. (Alvarez de Zayas, 1999) (Martí \& Perez, 1886) Es "...el sistema de acciones sucesivas y conscientes del hombre, que tiende a 
alcanzar un resultado que se corresponde con el objetivo trazado". (Salas Perea, 1999) (Urbina Laza, 2010)

Además de los métodos de enseñanza de la Didáctica General, o sea, los que abarcan los actos de interacción maestro-alumno, existen a la vez los métodos de enseñanza de las diferentes disciplinas, que no rebasan los marcos de los anteriores ya que se inscriben en ellos sin excepción, aunque pueden tener funciones que corresponden a un fin determinado. (Bravo Mancero \& Varguillas Carmona, 2015)

En toda ciencia aparecen los métodos mediante los cuales los sujetos se relacionan con el objeto y lo modifican, lo que permite dominar los conceptos, las leyes y los principios que caracterizan tanto al objeto, como a su movimiento.

Las ciencias clínicas también poseen un método: el método clínico. Ello significa que, para realizar su función de atención médica integral, el médico general integral básico cuenta con una serie de pasos que poseen una determinada secuencia. (Moreno Rodríguez, Deficiencias en la entrevista médica: un aspecto del método clínico, 2000) En los últimos años, en Cuba, varios autores han resaltado la importancia y plena vigencia de la utilización del método clínico en la práctica profesional. (Ilizástegui Dupuy \& Rodríguez Rivera, 1990) (Moreno Rodríguez, Crisis del método clínico, 1998)

Precisamente el método clínico es reconocido en documentos metodológicos rectores de la formación del médico general integral básico como el principal método de enseñanza en la mayoría de las asignaturas del ciclo clínico de la carrera de Medicina. 
En el folleto Orientaciones metodológicas sobre la educación en el trabajo, se plantea que en los tipos principales de la forma organizativa docente fundamental de la carrera de Medicina: la educación en el trabajo, se utiliza el método clínico para atender a los pacientes, por ser este el método científico cuando se trata de la atención médica a individuos. (Harrison, 1998) Textos básicos y de consulta de la carrera de Medicina describen y resaltan, de forma explícita o implícita, la importancia del método clínico en la asistencia a individuos enfermos. (Adams \& Víctor, 1982)

Se asume el planteamiento de (Alvarez de Zayas, 1999) quien señala que el método, como componente esencial de la ciencia, pasa a formar parte del contenido de la asignatura, como conocimiento y habilidad, condicionando este, en gran medida, el método de enseñanza. El citado autor añade que el método de la ciencia se traslada como habilidad al objetivo del proceso docente educativo; y se manifiesta, durante el desarrollo del proceso, en el método de enseñanza y aprendizaje. El estudiante para instruirse hará uso de los métodos propios de la ciencia incorporados al proceso docente como métodos de aprendizaje.

Cuando el médico general integral básico está desarrollando el método clínico en la atención a un paciente, emplea sus conocimientos para la identificación del problema de salud al poner en juego variadas habilidades profesionales, comunicativas y lógicas del pensamiento, en función del diagnóstico. Como subsiguiente paso en la ejecución del método clínico, también aplica los conocimientos relativos al tratamiento de los problemas identificados.

Al ser el método clínico el método de la profesión, el método que le permite al estudiante la interacción con su objeto de trabajo (en este caso el paciente) en la principal 
forma organizativa docente de las disciplinas y asignaturas de la profesión y, por tanto, de la carrera (la educación en el trabajo) constituye el método fundamental a través del cual se materializa, se concreta la actividad de aprendizaje del estudiante como proceso de construcción del conocimiento, formación de habilidades y hábitos y adquisición de valores. Es a través del método clínico donde se materializa también el proceso de comunicación entre el estudiante y el profesor, y a través del cual ambos, estudiantes y profesor, se relacionan con el objeto de aprendizaje; objeto que se personifica en el paciente. La actividad y la comunicación, junto a la motivación, son características inherentes al método de enseñanza, como componente del proceso docente educativo. (Adams \& Víctor, 1982)

Los estudios del método clínico accede a reconocer en los propios procedimientos que lo determinan como método de la profesión médica (interrogar, examinar, comparar, inducir, etc.) a los procedimientos didácticos que lo caracterizan como método de enseñanza; o sea, los procedimientos que caracterizan al método clínico se manifiestan como procedimientos didácticos en el proceso formativo. A los procedimientos que le son propios al método clínico se le añaden durante su utilización como método de enseñanza, otros procedimientos como son la demostración (que realiza el profesor, o los propios estudiantes en el seno del grupo), la explicación y la ejercitación.

Desde la perspectiva de los métodos de enseñanza de la Didáctica General y teniendo en cuenta el grado de participación de los sujetos en el proceso docente educativo, el método clínico puede ser utilizado como un método de elaboración conjunta (cuando estudiante y profesor interactúan en la atención a un paciente, por ejemplo) o como un método de trabajo independiente. Si el análisis se realiza en dependencia del nivel de dominio de la habilidad que se intente lograr en un momento determinado, y manipulando para ello la complejidad de los problemas de salud a resolver por el estudiante, el profesor puede utilizar el método clínico como un método reproductivo 
(aplicándolo en la solución de problemas sencillos, ya dominados por el estudiante) o como un método productivo (situaciones nuevas, casos más complejos).

\section{Conclusiones.}

La condición del método clínico como método de enseñanza en la carrera de Medicina significa que mediante este, y regulando el nivel de profundidad (complejidad) del objeto de aprendizaje en cada una de las disciplinas y asignaturas de la profesión, el estudiante aplica y logra el dominio de los conocimientos y la sistematización necesaria de las habilidades requeridas para intervenir sobre su objeto de trabajo, domina además la lógica de actuación de la profesión, el modo de actuación profesional.

De esta manera, con la utilización del método clínico como método de enseñanza, se evidencia que la lógica de la profesión se convierte en la lógica del proceso de formación del médico general integral básico.

\section{Bibliografía.}

Adams, R., \& Víctor, M. (1982). Cómo abordar al paciente neurológico. La Habana: CientíficoTécnica.

Alvarez de Zayas, C. (1999). La escuela en la vida: Didáctica. Ciudad de La Habana: Pueblo y Educación.

Bravo Mancero, P., \& Varguillas Carmona, C. (2015). Estrategias didácticas para la enseñanza de la asignatura Técnicas de Estudio en la Universidad Nacional de Chimborazo. Sophia, Colección de Filosofía de la Educación, 19(1), 271-290.

Dupuy F, I., \& Rodríguez Rivera, L. (1990). El método clínico. Rev Finlay, 4(4), 3-23.

Espinosa Brito, A. (2010). La unicidad en la diversidad. Factores en cuestión. MEDISUR, 8(5), 93103. 
Harrison, T. (1998). Principios de Medicina Interna (14 ed.). Madrid: Mc Graw-Hill Interamericana.

Ilizástegui Dupuy, F., \& Rodríguez Rivera, L. (1990). El método clínico. Educacion, 4(4), 3-23.

Martí, \& Perez, J. (1886). Trabajo Manual en las Escuelas. New York: La América.

Moreno Rodríguez, M. (1998). Crisis del método clínico. Rev Cubana Med, 37(1), 123-128.

Moreno Rodríguez, M. (2000). Deficiencias en la entrevista médica: un aspecto del método clínico. Rev Cubana Med, 39(1), 106-114.

Salas Perea, R. (1999). Educación en salud: Competencia y desempeño profesionales. La Habana: Editorial Ciencias Médicas.

Urbina Laza, O. (2010). Metodología para la evaluación de las competencias. Revista Cubana de Salud Pública; , 36(2), 165-174. 